\title{
Universal Torsion Induced Interaction from Large Extra Dimensions
}

\author{
Tatsu Takeuchi ${ }^{a}$, Lay Nam Chang ${ }^{a}$, Oleg Lebedev ${ }^{a}$, \\ and \\ Will Loinaz ${ }^{a, b}$ \\ ${ }^{a}$ IPPAP, Physics Department, Virginia Tech, Blacksburg, VA 24061 \\ ${ }^{b}$ Department of Physics, Amherst College, Amherst, MA 02001
}

\begin{abstract}
We consider a model with extra compact dimensions in which only gravity exists in the $4+n$ dimensional bulk. If the gravitational connection is not assumed to be symmetric, that is, unless torsion is artificially set to zero, then a universal contact interaction among the fermions on the 4-dimensional wall is induced. Using a global fit to $Z$-pole observables, we find the $3 \sigma$ bound on the scale of quantum gravity to be $M_{S}=28 \mathrm{TeV}$ for $n=2$. If Dirac or light sterile neutrinos are present, the data from SN1987A increase the bound to $\sqrt{n} M_{S} \geq 210 \mathrm{TeV}$.
\end{abstract}

Most theories of gravity incorporate the Poincaré group as a local symmetry. Some, like general relativity, achieve this by demanding local diffeomorphism invariance, with the additional requirement that gravity can be eliminated completely around a point by a coordinate choice. This strong form of the equivalence principle also restricts the resultant connection coefficient to be symmetric, thereby eliminating any reference to torsion in the consequent geometry. In effect, the theory "gauges" the translation group, with the energy-momentum tensor being the sole source of gravity.

The more general situation in which the Lorentz group is gauged as well will give rise to an antisymmetric part to the connection coefficient, the torsion tensor. This general situation obtains in the presence of intrinsic spin; the torsion tensor is then coupled to the intrinsic spin current, which then represents yet another source of gravity. Since this current cannot be eliminated by a choice of coordinates, the situation breaks the strong form of the equivalence principle. It is possible to require that the torsion tensor still vanishes, but this demand will need to be preserved under radiative corrections by invoking additional symmetries. Since the classic tests of general relativity do not involve intrinsic spin, the presence of torsion will not show up in any of these checks [1].

In this paper, we consider the consequences of torsion in the context of a model with large extra dimensions [2]. Gravity is allowed to exist in a $4+n$ dimensional 
bulk while all other fields are confined to a 4-dimensional wall. Allowing the torsion tensor $T_{\beta \gamma}^{\alpha}=\tilde{\Gamma}_{\beta \gamma}^{\alpha}-\tilde{\Gamma}_{\gamma \beta}^{\alpha}$ to be non-zero introduces an extra piece into the gravitational connection

$$
\tilde{\Gamma}_{\beta \gamma}^{\alpha}=\Gamma_{\beta \gamma}^{\alpha}+K_{\beta \gamma}^{\alpha},
$$

where $\Gamma_{\beta \gamma}^{\alpha}$ is the usual metric contribution, and $K_{\alpha \beta \gamma}=\frac{1}{2}\left(T_{\alpha \beta \gamma}-T_{\beta \alpha \gamma}-T_{\gamma \alpha \beta}\right)$ is known as the contorsion tensor. The action of our model is given by

$$
\begin{aligned}
& S=-\frac{1}{\hat{\kappa}^{2}} \int d^{4+n} x \sqrt{\left|\hat{g}_{4+n}\right|} \tilde{R} \\
& +\int d^{4} x \sqrt{\left|\hat{g}_{4}\right|} \frac{i}{2}\left[\bar{\Psi} \gamma^{\mu} \tilde{\nabla}_{\mu} \Psi-\left(\tilde{\nabla}_{\mu} \bar{\Psi}\right) \gamma^{\mu} \Psi+2 i M \bar{\Psi} \Psi\right] .
\end{aligned}
$$

Here $\hat{\kappa}^{2}=16 \pi G_{N}^{(4+n)}, \tilde{R}$ is the $4+n$ dimensional scalar curvature, and $\hat{g}_{4+n}$ and $\hat{g}_{4}$ are respectively the $4+n$ and 4 -dimensional (induced) metric determinants. The fermion $\Psi$ is coupled to gravity via the covariant derivative $\tilde{\nabla}_{\mu} \Psi=\partial_{\mu} \Psi+\frac{i}{2} \tilde{\omega}_{\mu}^{a b} \sigma_{a b} \Psi$, where $\tilde{\omega}_{\mu}^{a b}$ is the spin-connection, $\sigma_{a b}=\frac{i}{2}\left[\gamma_{a}, \gamma_{b}\right]$, with $a, b$ the local Lorentz indices. A general spin connection $\tilde{\omega}_{\mu}^{a b}$ can be expressed in terms of a torsion-free spinconnection $\omega_{\mu}^{a b}$, the contorsion tensor, and the vierbein $e_{\mu}^{a}$ :

$$
\tilde{\omega}_{\mu}^{a b}=\omega_{\mu}^{a b}+\frac{1}{4} K_{\lambda \mu}^{\nu}\left(e^{\lambda a} e_{\nu}^{b}-e^{\lambda b} e_{\nu}^{a}\right) .
$$

Elimination of torsion from the action by imposing the equations of motion results in [3]:

$$
\begin{aligned}
S= & -\frac{1}{\hat{\kappa}^{2}} \int d^{4+n} x \sqrt{\left|\hat{g}_{4+n}\right|} R \\
& +\int d^{4} x \sqrt{\left|\hat{g}_{4}\right|}\left[\bar{\Psi}\left(i \gamma^{\mu} \nabla_{\mu}-M\right) \Psi+\frac{3}{32} \frac{\sqrt{\left|\hat{g}_{4}\right|}}{\sqrt{\left|\hat{g}_{4+n}\right|}} \hat{\kappa}^{2}\left(\bar{\Psi} \gamma_{\mu} \gamma_{5} \Psi\right)^{2} \delta^{(n)}(0)\right] .
\end{aligned}
$$

where $R$ is the torsion-free curvature. The delta-function appearing in this expression should be regularized to account for a finite wall thickness:

$$
\delta^{(n)}(0) \rightarrow \frac{1}{(2 \pi)^{n}} \int_{0}^{M_{S}} d^{n} k=\frac{M_{S}^{n}}{2^{n-1} \pi^{n / 2} n \Gamma\left(\frac{n}{2}\right)},
$$

$M_{S}$ is the cutoff scale of the effective theory, here taken to be of the order of the inverse wall thinkness. The $4+n$ dimensional coupling constant $\hat{\kappa}$ is related to the 4-dimensional coupling $\kappa$ and the volume of the extra dimensions compactified on a torus via $\hat{\kappa}^{2}=\kappa^{2} V_{n}=16 \pi(4 \pi)^{n / 2} \Gamma(n / 2) M_{S}^{-(n+2)}[4]^{1}$. As a result, the leading $\mathcal{O}\left(\hat{\kappa}^{2}\right)$ torsion contribution to the action is given by

1) For simplicity we set the string scale and the $4+n$ dimensional Planck mass equal. 


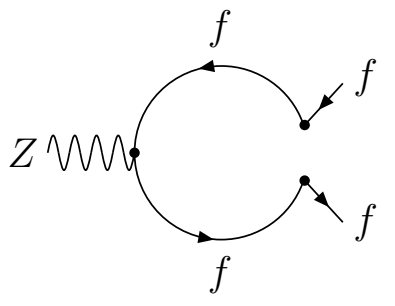

(a)

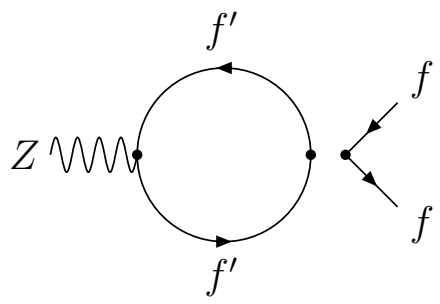

(b)

FIGURE 1. Corrections to the $Z f \bar{f}$ coupling from the universal contact interaction Eq. (6).

$$
\Delta S=\int d^{4} x \frac{3 \pi}{n M_{S}^{2}}\left[\sum_{j} \bar{\Psi}_{j} \gamma_{\mu} \gamma_{5} \Psi_{j}\right]^{2},
$$

where $j$ runs over all fermions existing on the wall. The expansion in $\hat{\kappa}$ is expected to be valid provided the typical energy $E$ of a physical process is below the cutoff scale $M_{S}$.

The torsion induced contact interaction Eq. (6) can be constrained through its effect on $Z$-pole electroweak observables. The corrections shown in Fig. 1 shifts the $Z$-couplings by

$$
\delta h_{L}=-\delta h_{R}=\frac{3 N_{c} m_{t}^{2}}{4 \pi n M_{S}^{2}} \ln \frac{M_{S}^{2}}{m_{t}^{2}},
$$

where the Z-vertex is defined as $-i \frac{g}{\cos \theta_{W}} Z_{\mu} \bar{\Psi} \gamma^{\mu}\left(P_{L} h_{L}+P_{R} h_{R}\right) \Psi$. Note that the contribution of torsion to $\delta h_{L}$ is strictly positive. Performing a global fit to the LEP/SLD electroweak observables will lead to a constraint on $\delta h_{L}$, which in turn will give us a limit on $M_{S}$. For $n=2$ we find the $3 \sigma$ bound to be

$$
M_{S} \geq 28 \mathrm{TeV} .
$$

For $n=4(6)$ the bound weakens to 19 (15) $\mathrm{TeV}$. The details of the analysis are presented elsewhere [3].

Other constraints are available as well. The universal $A \times A^{+}$contact interaction Eq. (6) affects at the tree level the differential cross sections for $e^{+} e^{-} \rightarrow f \bar{f}$ measured at LEP. The OPAL measurements [5] imply

$$
\sqrt{n} M_{S} \geq 10.3 \mathrm{TeV}
$$

at the $95 \%$ confidence level. Electron-quark contact interactions can also be constrained via HERA DIS data, Drell-Yan production at the Tevatron, etc. The global analysis [6] yields

$$
\sqrt{n} M_{S} \geq 5.3 \mathrm{TeV}
$$


Another potentially strong constraint can come from the measurement of the invisible width of the $\Upsilon$ and $J / \Psi$ resonances at B and $\tau$-charm factories [7].

A powerful astrophysical constraint can be derived if we admit existence of Dirac or light sterile neutrinos. For the case of Dirac neutrinos, the torsion-induced interaction containes a term

$$
\Delta \mathcal{L}=-\frac{6 \pi}{n M_{S}^{2}} \bar{q} \gamma^{\mu} \gamma_{5} q \bar{\nu}_{R} \gamma_{\mu} \nu_{R}
$$

This contact interaction provides a new channel of energy drain during neutron star collapse, since right handed neutrinos produced by nucleon interactions leave the core without rescattering This would affect neutron star evolution; in particular, it would modify the duration of the standard neutrino burst. From observations of SN 1987A one infers [8]

$$
\sqrt{n} M_{S} \geq 210 \mathrm{TeV}
$$

Similar considerations apply to the case of light sterile neutrinos: if $m_{\nu_{s}} \ll 50 \mathrm{MeV}$, the core temperature, the analysis is completely analogous to that of Dirac neutrinos and the bound (12) holds. This bound translates into an upper bound on the compactification radius of $3 \times 10^{-5} \mathrm{~mm}$ for $n=2$.

Since $M_{S}$ controls all gravity effects in extra dimensions, the limits on $M_{S}$ being larger than tens of $\mathrm{TeV}$ reported here imply weaker $\mathrm{KK}$ graviton couplings than those considered in the literature.

This research is supported in part by a grant from the U.S. Department of Energy, DE-FG05-92ER40709.

\section{REFERENCES}

1. I. Ciufolini, J. A. Wheeler, Gravitation and Inertia (Princeton University, New Jersey, 1995).

2. N. Arkani-Hamed, S. Dimopoulos and G. Dvali, Phys. Lett. B429, 263 (1998); Phys. Rev. D59, 086004 (1999); I. Antoniadis, N. Arkani-Hamed, S. Dimopoulos and G. Dvali, Phys. Lett. B436, 257 (1998).

3. L. N. Chang, O. Lebedev, W. Loinaz and T. Takeuchi, Phys. Rev. Lett. 85, 3765 (2000) [hep-ph/0005236].

4. T. Han, J. D. Lykken and R. Zhang, Phys. Rev. D59, 105006 (1999).

5. OPAL Collaboration, G. Alexander et al., Phys. Lett. B387, 432 (1996); G. Abbiendi et al., Eur. Phys. J. C6, 1 (1999).

6. K. Cheung, hep-ph/9807483; V. Barger, K. Cheung, K. Hagiwara, D. Zeppenfeld, Phys. Rev. D57, 391 (1998).

7. L. N. Chang, O. Lebedev and J. N. Ng, Phys. Lett. B441, 419 (1998) [hep$\mathrm{ph} / 9806487$.

8. J. A. Grifols, E. Massó and R. Toldrà, Phys. Rev. D57, 2005 (1998) [hep-ph/9707531]. 